\title{
INVARIANT SUBSPACES FOR ALGEBRAS OF SUBNORMAL OPERATORS
}

JAMES E. THOMSON ${ }^{1}$

\begin{abstract}
Every rationally cyclic subnormal operator has a hyperinvariant subspace.
\end{abstract}

A bounded linear operator on a Hilbert space is defined to be subnormal if it is the restriction to an invariant subspace of a bounded normal operator. S. W. Brown [4] proved that every subnormal operator has a nontrivial invariant subspace. An idea in his proof leads to a stronger theorem, whose proof is easier.

THEOREM. Let $\mu$ be a compactly supported positive measure on the complex plane $\mathbf{C}$. Let $H$ be a closed subspace of $L^{2}(\mu)$ with $1 \in H$. Let $A$ be a subalgebra of $L^{\infty}(\mu)$ containing the function $z$ such that $A H \subset H$. Then there exists a nontrivial closed subspace $K$ of $H$ such that $A K \subset K$.

Before proving the theorem, we will point out some consequences. A subnormal operator $S$ on a space $H$ is rationally cyclic if there exists a vector $x$ in $H$ such that the set $\{r(S) x: r \in \operatorname{Rat}(\sigma(S))\}$ is dense in $H$, where $\operatorname{Rat}(\sigma(S))$ denotes the algebra of rational functions with poles off the spectrum of $S$.

For each such $S$, there exists a measure $\mu$ such that $S$ is unitarily equivalent to multiplication by $z$ on $R^{2}(\sigma(S), \mu)$, the closure in $L^{2}(\mu)$ of $\operatorname{Rat}(\sigma(S))$ [5, p. 146]. Under this representation each operator that commutes with $S$ is represented by multiplication by a function in $R^{2}(\sigma(S), \mu) \cap L^{\infty}(\mu)$, and conversely [5, p. 147]. A subspace invariant for every operator that commutes with $S$ is called hyperinvariant. If we let $H=R^{2}(\sigma(S), \mu)$ and $A=R^{2}(\sigma(S), \mu) \cap L^{\infty}(\mu)$ in the theorem, then we obtain a hyperinvariant subspace.

COROLLARY 1. Every rationally cyclic subnormal operator has a hyperinvariant subspace.

The following is a trivial consequence of Corollary 1.

COROLlARY 2. Every subnormal operator $S$ has a subspace invariant for the algebra $\{r(S): r \in \operatorname{Rat}(\sigma(S))\}$.

Received by the editors April 7, 1985.

1980 Mathematics Subject Classification. Primary 47B20.

Kel words and phrases. Subnormal operators, invariant subspaces.

${ }^{1}$ Research supported in part by a grant from the National Science Foundation.

1986 American Mathematical Society $0002-9939 / 86 \$ 1.00+\$ .25$ per page 
An excellent general reference for subnormal operators is the book by J. B. Conway [5]. J. E. Brennan [1-3] has worked extensively on rationally invariant subspaces, and the author has found that work instructive.

Proof of The Theorem. If $H=L^{2}(\mu)$, then let $K=f H$, where $f$ is a nontrivial characteristic function.

Assume $H \neq L^{2}(\mu)$. Then there exists a nonzero function $g$ in $L^{2}(\mu)$ such that $\int f g d \mu=0$ for every $f$ in $H$. Let $p=3$ and $q=3 / 2$ for the remainder of this paper. By [1] and [5, p. 316] there exists a point $w$ in $\mathbf{C}$ such that $g(z)(z-w)^{-1}$ is in $L^{q}(\mu), \mu(\{w\})=0$ and

$$
\int g(z)(z-w)^{-1} d \mu(z) \neq 0
$$

Fix such a point $w$.

Let $A^{p}(\mu)$ denote the closure in $L^{p}(\mu)$ of $A$. Note that $A^{p}(\mu) \subset H$. Define a bounded linear functional $L$ on $A^{p}(\mu)$ by

$$
L(f)=\int f(z) g(z)(z-w)^{-1} d \mu(z)
$$

for $f$ in $A^{p}(\mu)$. By the Hahn-Banach theorem there exists a norm-preserving extension of $L$ to $L^{p}(\mu)$. This extension is represented by a function $h$ in $L^{q}(\mu)$ with $\|h\|_{q}=\|L\|$. That is,

$$
L(f)=\int f h d \mu
$$

for each $f$ in $A^{p}(\mu)$. Since the closed unit ball of $A^{p}(\mu)$ is weakly compact, there exists a function $r$ in $A^{p}(\mu)$ with $\|r\|_{p}=1$ and $L(r)=\|L\|$. Thus,

$$
\|h\|_{q}=\|L\|=L(r)=\int r h d \mu \leqslant\|r\|_{p}\|h\|_{q}=\|h\|_{q}
$$

The equality in Holder's inequality above implies that

$$
|r|^{p}=a|h|^{q} \text { a.e. }(\mu)
$$

for some positive constant $a$, or

$$
|r|^{2}=b|h| \text { a.e. }(\mu)
$$

for some positive constant $b$.

Let $x=h / r$ on the set where $r$ is nonzero, and zero elsewhere. The function $x$ is in $L^{2}(\mu)$ because

$$
\int|x|^{2} d \mu=\int|h|^{2}|r|^{-2} d \mu=b^{-1} \int|h| d \mu<\infty .
$$


Let $K$ be the closure of the linear manifold $(z-w) A r$. Clearly $A K \subset K$. For each $f$ in $A$,

$$
\begin{aligned}
\int(z-w) f r x d \mu & =\int(z-w) f h d \mu \\
& =\int(z-w) f(z) g(z)(z-w)^{-1} d \mu(z) \\
& =\int f g d \mu=0 .
\end{aligned}
$$

But $r$ is in $H$ and

$$
\int r x d \mu=\int h d \mu=\int g(z)(z-w)^{-1} d \mu(z) \neq 0 .
$$

Thus $K$ is a proper subspace of $H$.

Remark. In the proof above, if $A$ is the algebra of polynomials (similarly for rational functions), then there exists a constant $c$ such that $f(w)=(f r, c \bar{x})$ for every polynomial $f$, where (, ) denotes the standard inner product on $L^{2}(\mu)$. The idea of using factorization to obtain point evaluation is due to Brown [4]. The fact that there are $L^{p}(\mu)$-continuous point evaluations is due to Brennan [1]. The original contribution of this paper is the method for combining those ideas.

\section{REFERENCES}

1. J. E. Brennan, Invariant subspaces and rational approximation, J. Funct. Anal. 7 (1971), 285-310.

2. Invariant subspaces and weighted polynomial approximation, Ark. Mat. 11 (1973), 167-189.

3. Point evaluations, invariant subspaces and approximation in the mean by polynomials, $\mathrm{J}$. Funct. Anal. 34 (1979), 407-420.

4. S. W. Brown, Some invariant subspaces for subnormal operators, Integral Equations Operator Theory 1 (1978), 310-333.

5. J. B. Conway, Subnormal operators, Pitman, Boston, Mass., 1981.

Department of Mathematics, Virginia Polytechnic Institute and State University, BlacksBURG, VIRGINIA 24061 